\title{
Should treatment effects be estimated in pilot and feasibility studies?
}

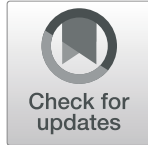

Julius Sime

\begin{abstract}
Background: Feasibility studies and external pilot studies are used increasingly to inform planning decisions related to a definitive randomized controlled trial. These studies can provide information on process measures, such as consent rates, treatment fidelity and compliance, and methods of outcome measurement. Additionally, they can provide initial parameter estimates for a sample size calculation, such as a standard deviation or the 'success' rate for a binary outcome in the control group. However, the issue of estimating treatment effects in pilot or feasibility studies is controversial.

Methodological discussion: Between-group estimates of treatment effect from pilot studies are sometimes used to calculate the sample size for a main trial, alongside estimated standard deviations. However, whilst estimating a standard deviation is an empirical matter, a targeted treatment effect should be established in terms of clinical judgement, as a minimum important difference (MID), not through analysis of pilot data. Secondly, between-group effects measured in pilot studies are sometimes used to indicate the magnitude of an effect that might be obtained in a main trial, and a decision on progression made with reference to the associated confidence interval. Such estimates will be imprecise in typically small pilot studies and therefore do not allow a robust decision on a main trial; both a decision to proceed and a decision not to proceed may be made too readily. Thirdly, a withingroup change might be estimated from a pilot or a feasibility study in a desire to assess the potential efficacy of a novel intervention prior to testing it in a main trial, but again such estimates are liable to be imprecise and do not allow sound causal inferences.

Conclusion: Treatment effects calculated from pilot or feasibility studies should not be the basis of a sample size calculation for a main trial, as the MID to be detected should be based primarily on clinical judgement rather than statistics. Deciding on progression to a main trial based on these treatment effects is also misguided, as they will normally be imprecise, and may be biased if the pilot or feasibility study is unrepresentative of the main trial.
\end{abstract}

Keywords: Pilot studies, Feasibility studies, Treatment effects, Randomized controlled trials

\section{Background}

Feasibility studies and external pilot studies are increasingly common precursors to a main, or definitive, randomized controlled trial $[1,2]$. These studies may address process measures, such as the number of eligible patients in a centre, the consent rate, rates of treatment fidelity and compliance, and the methods of randomization, blinding and outcome measurement $[3,4]$. They may also function to estimate parameters required for a sample size calculation, such as the standard deviation of a continuous outcome measure $[5,6]$, or possibly the control group proportion for a binary

\section{Correspondence: j.sim@keele.ac.uk}

School of Primary, Community and Social Care and Keele Clinical Trials Unit, Keele University, Staffordshire ST5 5BG, UK outcome. In principle, the intracluster correlation coefficient could be estimated in the case of a cluster trial, though this is subject to important caveats regarding sample size [7].

A pilot study is considered to be a version of the main trial (or possibly part thereof) run on a smaller scale in order to determine whether its components work effectively together, and thus normally involves randomization (or at least allocation) to two or more groups, whereas a feasibility study may be a single-group study and need not adopt the design of the intended main trial $[8,9]$. Both pilot and feasibility studies can in principle be used to estimate a treatment effect, though the CONSORT extension for such studies does not recommend formal hypothesis testing of such effects [10]. In a pilot study, both a

(c) The Author(s). 2019 Open Access This article is distributed under the terms of the Creative Commons Attribution 4.0 International License (http://creativecommons.org/licenses/by/4.0/), which permits unrestricted use, distribution, and 
within- and a between-group estimate can be obtained, but in a single-group feasibility study, only a within-group estimate is possible. The motivation for such estimates may be to determine an effect that may be either important and/or realistic for a main trial [11]. Deriving these measures of effect may seem attractive, in an attempt to maximize the information generated by the pilot or feasibility study. In practice, however, producing such estimates is less straightforward and may be misguided.

\section{Common misuses of between-group estimates of treatment effect \\ Estimating the minimum important difference for a sample size calculation}

Reviews suggest that researchers sometimes use a betweengroup estimate of effect (or a standardized effect size) from a pilot or feasibility study as the basis of a sample size calculation for a main trial [12-15]. For example, Uszynski et al. [16] and Kemp et al. [17] used observed between-group differences (expressed as standardized effect sizes) and Mollart et al. [18] used observed between-group differences in proportions to determine the sample size required for a subsequent main trial. If this strategy is adopted in order to determine an important effect on which the sample size calculation should be based, it seems ill-founded. An effect such as a mean difference estimated from a pilot study may give some indication of the effect that may be found. However, it does not indicate the effect that is worth finding, which needs to be informed by clinical judgement and patient perspectives, and expressed as an a priori minimum important difference (MID) - one sufficiently large to affect clinical decision-making $[3,19]$. Moreover, basing the sample size for a continuous outcome measure on a standardized effect size is uninformative, as it confounds the mean difference with its standard deviation, focusing attention away from the absolute magnitude of the difference. It also further obscures the important distinction that, for sample size purposes, the standard deviation needs to be calculated but the magnitude of the difference needs to be specified. A further problem is that any such effect from a pilot study of typical size will often be too imprecise to provide useful guidance for a sample size calculation $[19,20]$.

\section{Using the MID to decide whether to proceed to main trial}

Another possible purpose of estimating a between-group treatment effect is to gain an indication as to whether an MID, determined a priori, might feasibly be obtained in the main trial, and thereby inform a decision as to whether or not to undertake the main trial. However, the criterion on which such a decision should be taken is unclear. A straightforward approach is simply to produce a point estimate from the pilot study and compare this with the MID or other predetermined cut-off, such that if the point estimate is at least as large as the MID the main study could proceed. For example, Ruzicka et al. [21] have proposed for their pilot study that if at least $15 \%$ of participants exhibit a positive response to the intervention tested, a full trial would be warranted. However, at their intended sample size of 40, a 95\% confidence interval (CI) around a point estimate of $15 \%$ would extend from approximately 7 to $29 \%$ (using the Wilson method), indicating a very imprecise estimate on which to base a decision regarding progression to a main trial.

Westlund and Stuart [22] have shown through simulations that this imprecision also leads to inappropriate decisions to proceed or not to proceed to a main trial, if the point estimate from the pilot study either overestimates or underestimates, respectively, the MID. Table 1 shows the percentages of simulated pilot studies in which a decision to proceed to a main trial would be taken on the criterion of the point estimate exceeding the MID (assumed to be a standardized effect of 0.25). For studies estimating a true effect lower than the MID, a mistaken decision to proceed to a main trial would be taken in $19 \%$ and $43 \%$ of studies for true standardized effects of 0.00 and 0.20 , respectively. Conversely, mistaken decisions not to proceed would be taken in $19 \%$ and $3 \%$ of studies for true standardized effects of 0.50 and 0.80 , respectively.

\section{Using a confidence interval around a between-group effect to decide whether to proceed to main trial}

Another possibility is to construct a CI for the betweengroup effect-here, a mean difference will be assumedand observe where the MID lies in relation to this CI [23]. If the lower bound of the CI lies above the MID, all plausible values of the true treatment effect would be at least as large as the MID. One can therefore be reassured, at the appropriate level of confidence, that at an effect of at least the MID will be observed in the main trial (the same interpretation could be made if the lower bound happened to lie precisely on the MID). However, as noted earlier, the likely small size of the pilot study would produce a wide $\mathrm{CI}$, making it hard to exclude the MID from the CI, and thus with little probability that the main trial would be implemented. Moreover, a judgement on a CI in terms of the inclusion or exclusion of a particular value is equivalent to a hypothesis test (at a 5\% significance level for a 95\% CI), which could thereby prejudge the conclusion of the main trial. For example, if the $\mathrm{CI}$ included the null value of the treatment effect this would serve to reject the alternative hypothesis that would be tested in the main trial-but misleadingly, given that the chance of a type 2 error (false negative) would be unacceptably high in a typical pilot study.

An alternative, less stringent criterion, described by Arnold et al. [24], would be satisfied if the MID lies within (rather than necessarily below) the CI, suggesting that the MID is among the plausible values of the effect 
Table 1 Percentage of simulated pilot studies, estimating differing true standardized effect sizes, in which a main trial would be favoured when the observed point estimate exceeded the minimum important difference (0.25). One hundred thousand simulations were performed for each scenario [22]

\begin{tabular}{llllll}
\hline & \multicolumn{2}{l}{ True standardized effect size } & & \\
\cline { 2 - 3 } \cline { 6 - 6 } & Below MID & & & Above MID \\
\cline { 2 - 3 } & 0.00 & 0.20 & & 0.50 & 0.80 \\
\hline Pilot studies (\%) indicating progression to main trial & 19 & 43 & & 81 & 97 \\
\hline
\end{tabular}

that would be achieved in the main trial. Lee et al. [23] add the requirement that the point estimate of the treatment effect should be greater than zero (or, equivalently, below zero if a negative effect is of interest). However, in this approach, unless the MID lies at or very close to the lower bound of the $\mathrm{CI}$, a range of other values, smaller than the MID, would also be plausible estimates of the effect to be observed in the main trial. Furthermore, if the point estimate was smaller than the MID, this method could have the inappropriate consequence that the smaller the pilot study-and hence the wider the CI-the more likely it is that the MID would be captured by the upper bound of the CI, and a decision to undertake the main trial thereby endorsed.

This method of assessing the MID was used in a pilot study $(n=12)$ of splinting for spasticity in stroke survivors [25]. The assumed MID, expressed as a between-group difference in percentage improvement, was $40 \%$ for each outcome. On the basis of four outcomes for which a $95 \%$ CI included this MID, progression to a main trial was considered to be warranted. As Fig. 1 demonstrates, however, the wide CIs show the imprecision of these estimates, and in the case of three of the outcomes (A, B and C), they indicate the very wide range of alternative values below the MID also included within the CI.

Both when requiring the MID to lie below the $\mathrm{CI}$ and when requiring it to lie within it, the width of the $\mathrm{CI}$ for a continuous outcome, and hence the decision made regarding the MID, will depend upon the standard deviation of the treatment effect. In a small pilot study, this will be estimated imprecisely. Lack of precision is thus problematic for any formal decisions made regarding a main trial.

\section{Simulated example}

Figure 2 shows confidence intervals calculated on simulated data for 20 pilot studies-of a typical size of $n=34$ [12]-each estimating a mean difference of 10 for the unknown true treatment effect, with a standard deviation of 20. The MID is assumed to be 4 . If progression to a main study were determined on the basis of the lower bound of the CI lying above the MID, this decision would be negative in $9(4,7,8,9,13,14,16,18,19)$ of the simulated pilot studies and affirmative in the other 11. As only one pilot study would actually be performed, this illustrates how the decision regarding progression to the main trial would be very much at the mercy of sampling variability. It can also be noted that if the criterion were switched to that of including a value of at least the MID within the $\mathrm{CI}$, all of the studies would favour the main trial. However, in six of the studies $(7,8,13,14,16)$, a wide range of effects lower than the MID would be plausible alternative estimates of the true treatment effect. Which of the two interpretations of the $\mathrm{CI}$ is chosen clearly has a marked effect on the likelihood of recommending the main trial. Furthermore, in two of the studies (12 and 19), where the lower bound of the $\mathrm{CI}$ lies extremely close to the MID, if the criterion were that of requiring the MID to lie below the $\mathrm{CI}$ a once-and-for-all decision on a main trial would be made on the basis of a very small margin. Small arbitrary changes in the sample size of these two studies (two fewer participants in study 12 or two additional participants in study 19) would reverse the decision.

Thus, in a typically small pilot study, generating a wide $\mathrm{CI}$, if a judgement is made on the basis of the MID lying within the $\mathrm{CI}$, a decision to proceed to the main trial might be made too readily because values smaller than the MID are also likely to be included in the CI, and would therefore be plausible alternative values of the treatment effect to be found in the main trial. Conversely, when the MID is required to lie below the CI, a decision either to proceed or not to proceed with the main trial might be made too readily owing to random sampling variability affecting the lower bound of the CI. In both cases, a definitive decision would be made on proceeding to a main trial-which would, if conducted, gather robust evidence-on the comparatively scant evidence produced by a pilot study.

It has been suggested that a range of confidence levels, including and extending below 95\%, could be used to evaluate treatment effects from a pilot study [23]; the largest confidence level at which the MID is excluded from the CI represents the degree to which the researcher is reassured of attaining the MID in the main trial. This method is shown in Fig. 3 for study 18 in the simulation. The highest confidence level at which the MID is excluded from the CI is $75 \%$, and it is therefore with a corresponding degree of reassurance that progression to the main trial could be recommended. Whilst the focus on varying levels of confidence allows a more considered and less automatic decision on progression 


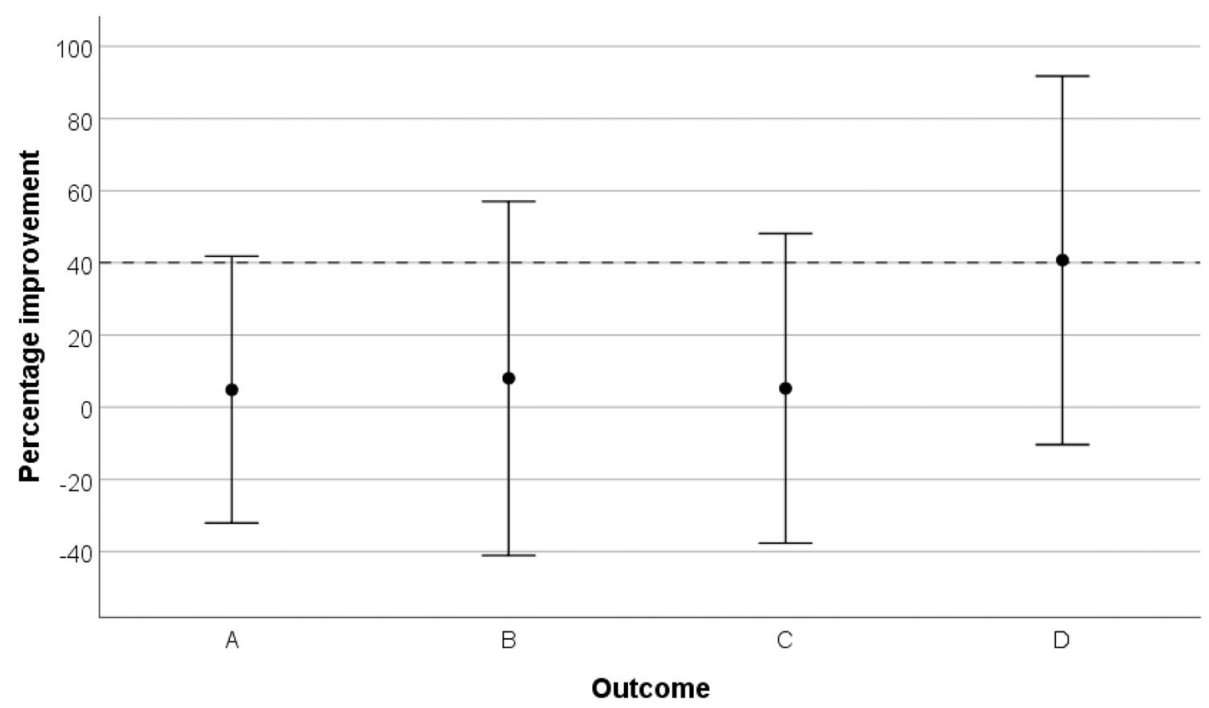

Fig. 1 Estimated treatment effects, with 95\% confidence intervals, reported for four outcomes by Sheehan et al. [25]. A difference in percentage improvement of $40 \%$ (indicated by the dashed horizontal reference line) was taken to be the minimum important difference

to a main trial than the previous approaches, this method is still vulnerable to sampling imprecision.

A further difficulty, common to each of the above strategies, is that unless an external pilot study is run in exactly the same way as the proposed main trial-in the same clinical population, in comparable centres, with equivalent strategies to prevent bias or confounding, with a similar level of compliance with the study protocol, and with the same covariates in the analysis-the estimate from the pilot study may be systematically biased, and thereby an unreliable indicator of what might occur in the main trial. Biased estimates may also arise in a small pilot study through substantial random baseline imbalance (referred to as 'chance bias' [26]). For example, in a randomized pilot study of treatments for Achilles tendon pain ( $n=8$ in each of two groups), Chester et al. [27] noted marked baseline differences between groups in sex (4 males versus 7 males), additional pathologies (6 versus 2) and mean duration of symptoms (23 months versus 14 months).

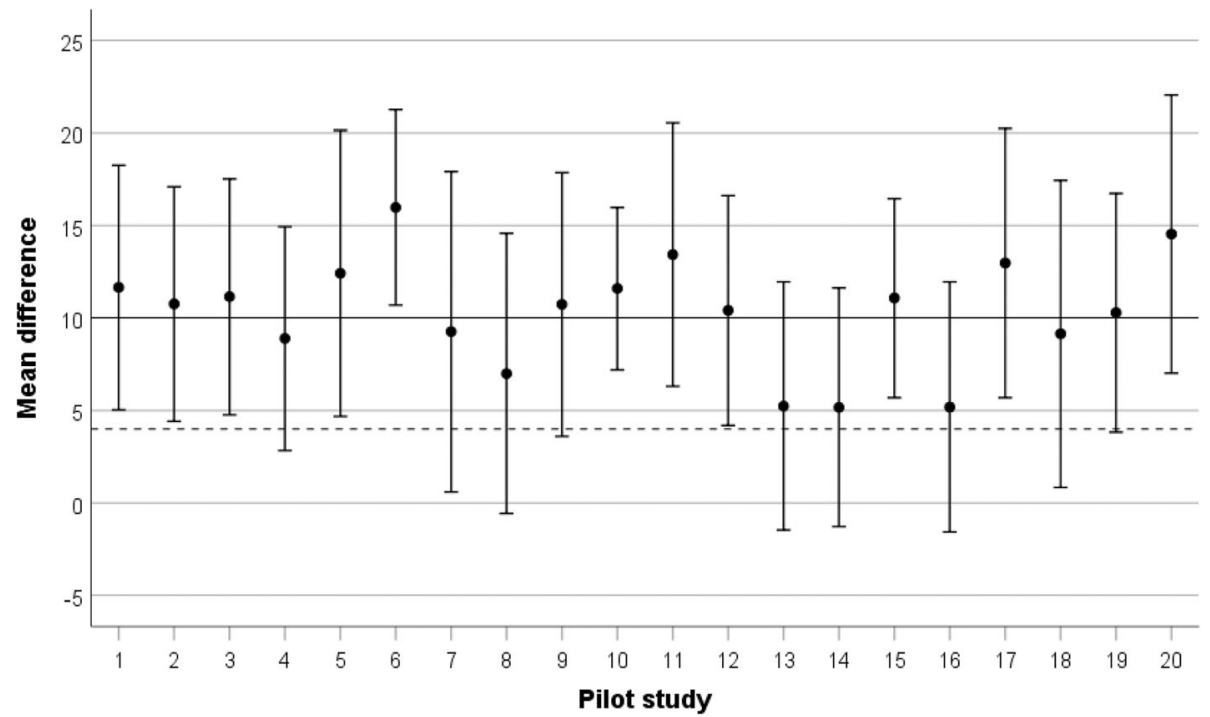

Fig. 2 Simulated data for 20 pilot studies (each $n=34$ ) estimating an unknown 'true' treatment effect of 10, with a standard deviation of 20, and with an assumed minimum important difference of 4 (dashed horizontal reference line). Error bars are $95 \%$ confidence intervals, and the observed underlying standard deviations range from 12.6 to 24.8 
A final problem arises in respect of point estimates of between-group effects if these are used both to decide on progression to a main trial and to determine its sample size $[19,22]$. In the first case, a decision to proceed to the main trial is only likely to be made if the estimate from the pilot study appears to be greater than or equal to the MID; estimates below the MID are clearly very unlikely to support progression to a main trial. Thus, trials that are recommended on this basis are liable to be associated with pilot studies that have overestimated the true MID. As sample size calculations would likely only be performed when the observed treatment effect favoured a main trial in this way, these calculations are in turn liable to be based on overestimates of the true MID, resulting in a main study that would be underpowered [19, 22].

\section{Within-group estimates of treatment effect}

Sometimes, a pilot or feasibility study might analyse within-group effects as a basis for recommending a future trial. For example, Garcia et al. [28] and Galantino et al. [29] derived such estimates from single-group feasibility studies of acupuncture for cancer pain $(n=51)$, and tai chi for breast cancer $(n=12)$, respectively. One reason why a trialist might wish to use a pilot or feasibility study in this way is to seek reassurance that a new untested intervention is effective in its own right, before proceeding to compare it with standard therapy or placebo (though, importantly, a single-group analysis such as this would not permit a robust causal inference as to the effect of the intervention on the outcome).

In such an approach, a CI might be constructed around a within-group estimate of effect for the new intervention, in relation to a minimum clinically important effect-in this case, a minimum important change (MIC) - and interpreted in a similar way to a between-group effect (though in the two examples given [28, 29], no CIs were presented and no MICs were cited). This approach would not encourage an inappropriate judgement as to the conclusion of the main trial, in the way that estimating a between-group effect might, as it is answering a different question-one of absolute rather than relative effectiveness. However, it faces the same difficulties in terms of how to interpret the relationship between the MIC and the $\mathrm{CI}$, and is subject to similar problems of sampling imprecision.

\section{Conclusion}

On the basis that it is normally unwise simply to ignore information, trialists may wish to calculate and examine estimates of treatment effect from pilot or feasibility studies, to gain some informal reassurance (or not) of what might be expected in the main trial. However, if the size of the pilot or feasibility study has not been formally calculated to provide an appropriate level of precision, these estimates are based on relatively meagre evidence and are therefore unreliable, and may result in inappropriate decisions either to proceed or not to proceed to a main trial. Moreover, it is not clear how they should most appropriately be interpreted. Such estimates should not therefore play a part in any formal decision-making regarding progression to a main trial, unless perhaps combined with other prior robust information, such as in a Bayesian decision model $[23,30]$. Moreover, treatment effects calculated from pilot studies should not be among the parameter estimates used in sample size calculations for a main trial, as the targeted treatment effect is predominantly a matter of judgement,

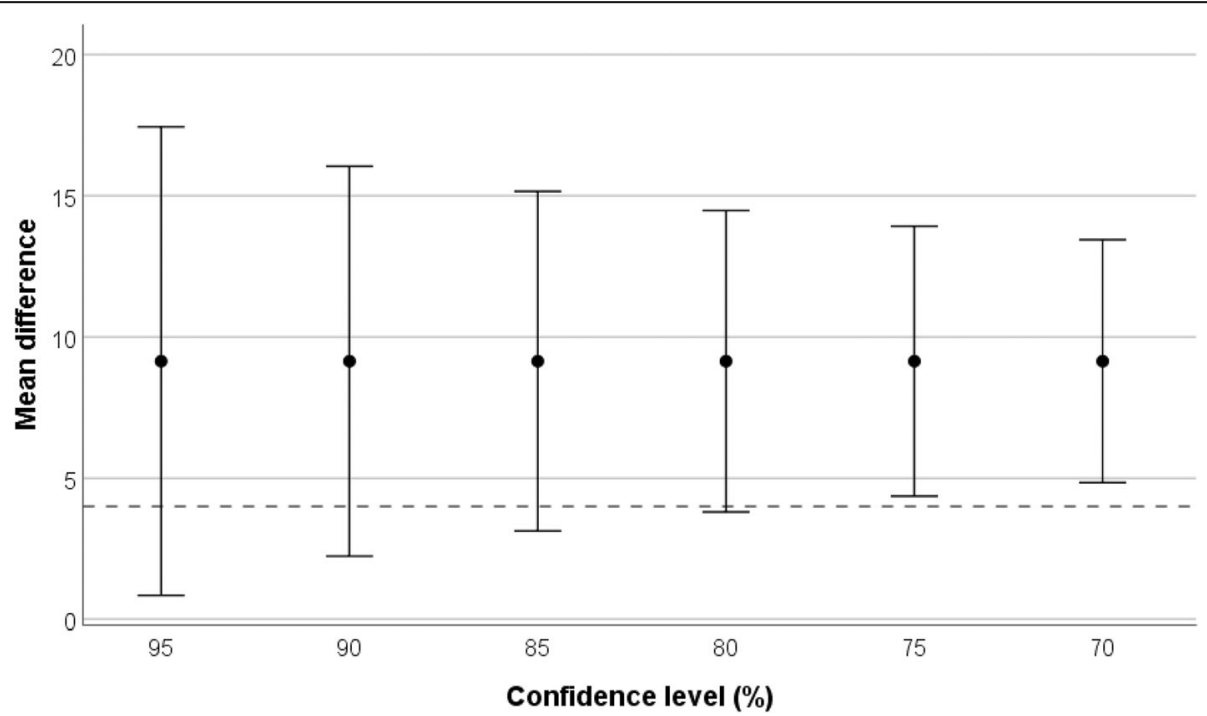

Fig. 3 Confidence intervals for the estimated treatment effect in study $18(n=34)$ in the simulated example, at varying confidence levels from 95 to $70 \%$. The dashed horizontal reference line indicates the minimum important difference 
not statistics; the effect one might detect does not determine the effect one needs to detect. Estimates of treatment effect derived from pilot and feasibility studies therefore provide information of very limited value and may do more to mislead than to enlighten.

It might be argued that the imprecision that results from small samples also affects other parameters that might be estimated in a pilot or feasibility study, such as a standard deviation. This is true, but not all such estimates are the basis of a largely irrevocable decision such as whether or not to proceed to a definitive study. A sample size based on an estimated standard deviation can often be revised in the light of further estimates from accruing data [31], and as the specific concern is a possible underestimation of the standard deviation, this can be at least partially offset by applying an inflation factor to the estimate [5].

Finally, it should be noted that although the focus has been on external pilot studies, key issues such as the imprecision of estimates of treatment effect, and the difficulties in assessing them in relation to decisions on progression or continuation to the main trial, are also relevant to internal pilot studies.

\section{Abbreviations}

Cl: Confidence interval; MIC: Minimum important change; MID: Minimum important difference

\section{Acknowledgements}

The author is grateful to Professor Gillian Lancaster and Professor Jackie Waterfield for helpful comments on a draft of this article.

\section{Authors' contributions}

The author conceived, wrote and approved the final manuscript.

\section{Funding}

No funding supported this work.

\section{Availability of data and materials}

Not applicable

\section{Ethics approval and consent to participate}

Not applicable

\section{Consent for publication}

Not applicable

\section{Competing interests}

The author declares that he has no competing interests

Received: 6 July 2019 Accepted: 21 August 2019

Published online: 28 August 2019

\section{References}

1. Thabane L, Ma J, Chu R, Cheng J, Ismaila A, Rios LP, Robson R, Thabane M, Giangregorio L, Goldsmith CH. A tutorial on pilot studies: the what, why and how. BMC Med Res Methodol. 2010;10:1. https://doi.org/10.1186/1471-2288-10-1.

2. Lancaster GA, Dodd S, Williamson PR. Design and analysis of pilot studies: recommendations for good practice. J Eval Clin Pract. 2004;10(2):307-12. https://doi.org/10.1111/j.2002.384.doc.x.

3. Leon $A C$, Davis $L L$, Kraemer HC. The role and interpretation of pilot studies in clinical research. J Psychiatr Res. 2011;45(5):626-9. https://doi.org/10.1016/ j.jpsychires.2010.10.008

4. Abbott JH. The distinction between randomized clinical trials (RCTs) and preliminary feasibility and pilot studies: what they are and are not. J
Orthop Sports Phys Ther. 2014;44(8):555-8. https://doi.org/10.2519/ jospt.2014.0110

5. Sim J, Lewis M. The size of a pilot study for a clinical trial should be calculated in relation to considerations of precision and efficiency. J Clin Epidemiol. 2012;65(3):301-8. https://doi.org/10.1016/j.jclinepi.2011.07.011.

6. Whitehead AL, Julious SA, Cooper CL, Campbell MJ. Estimating the sample size for a pilot randomised trial to minimise the overall trial sample size for the external pilot and main trial for a continuous outcome variable. Stat Methods Med Res. 2016;25(3):1057-73. https:// doi.org/10.1177/0962280215588241.

7. Eldridge SM, Costelloe CE, Kahan BC, Lancaster GA, Kerry SM. How big should the pilot study for my cluster randomised trial be? Stat Methods Med Res. 2016;25(3):1039-56. https://doi.org/10.1177/0962280215588242.

8. Arain M, Campbell MJ, Cooper CL, Lancaster GA. What is a pilot or feasibility study? A review of current practice and editorial policy. BMC Med Res Methodol. 2010;10:67. https://doi.org/10.1186/1471-2288-10-67.

9. Eldridge SM, Lancaster GA, Campbell MJ, Thabana L, Hopewell S, Coleman $\mathrm{CL}$, Bond CM. Defining feasibility and pilot studies in preparation for randomised controlled trials: development of a conceptual framework. PLoS One. 2016;11(3):e0150205. https://doi.org/10.1371/journal.pone.0150205.

10. Eldridge SM, Chan CL, Campbell MJ, Bond CM, Hopewell S, Thabane L, Lancaster GA. and on behalf of the PAFS consensus group. CONSORT 2010 statement: extension to randomised pilot and feasibility trials. Pilot Feasibility Stud. 2016;2:64. https://doi.org/10.1186/s40814-016-0105-8.

11. Cook JA, Julious SA, Sones W, Hampson LV, Hewitt C, Berlin JA, Ashby D, Emsley R, Fergusson DA, Walters SJ, Wilson ECF, Maclennan G, Stallard N, Rothwell JC, Bland M, Brown L, Ramsay CR, Cook A, Armstrong D, Altman D, Vale LD. DELTA2 guidance on choosing the target difference and undertaking and reporting the sample size calculation for a randomised controlled trial. Trials. 2018;19(1):606. https://doi.org/10.1186/s13063-018-2884-0.

12. Shanyinde M, Pickering RM, Weatherall M. Questions asked and answered in pilot and feasibility randomized controlled trials. BMC Med Res Methodol. 2010;11:117. https://doi.org/10.1186/1471-2288-11-117.

13. Hislop J, Adewuyi TE, Vale LD, Harrild K, Fraser C, Gurung T, Altman DG, Briggs AH, Fayers P, Ramsay CR, Norrie JD, Harvey IM, Buckley B, Cook JA, DELTA group. Methods for specifying the target difference in a randomised controlled trial: the Difference ELicitation in TriAls (DELTA) systematic review. PloS Med. 2014;11(5):e1001645. https://doi.org/10.13 71/journal.pmed. 1001645 .

14. Abdulatif M, Mukhtar A, Obayah G. Pitfalls in reporting sample size calculation in randomized controlled trials published in leading anaesthesia journals: a systematic review. Br J Anaesth. 2015;115(5):699-707. https://doi. org/10.1093/bja/aev166.

15. Kaur N, Figueiredo S, Bouchard V, Moriello C, Mayo N. Where have all the pilot studies gone? A follow-up on 30 years of pilot studies in Clinical Rehabilitation. Clin Rehabil. 2017;31(9):1238-48. https://doi.org/1 $0.1177 / 0269215517692129$.

16. Uszynski MK, Purtill H, Donnelly A, Coote S. Comparing the effects of wholebody vibration to standard exercise in ambulatory people with multiple sclerosis: a randomised controlled feasibility study. Clin Rehabil. 2016;30(7): 657-68. https://doi.org/10.1177/0269215515595522.

17. Kemp JL, Coburn SL, Jones DM, Crossley KM. The Physiotherapy for Femoroacetabular Impingement Rehabilitation STudy (physioFIRST): a pilot randomized controlled trial. J Orthop Sports Phys Ther. 2018:48(4):307-15. https://doi.org/10.2519/jospt.2018.7941.

18. Mollart L, Skinner V, Foureur M. A feasibility randomised controlled trial of acupressure to assist spontaneous labour for primigravid women experiencing a post-date pregnancy. Midwifery. 2016;36:21-7. https://doi. org/10.1016/j.midw.2016.02.020.

19. Kraemer HC, Mintz J, Noda A, Tinklenberg J, Yesavage JA. Caution regarding the use of pilot studies to guide power calculations for study proposals. Arch Gen Psychiatry. 2006;63(5):484-9. https://doi.org/10.1001/ archpsyc.63.5.484.

20. Friedman LM, Furberg CD, DeMets DL, Reboussin DM, Granger CB. Fundamentals of clinical trials. 5th ed. Heidelberg: Springer; 2015.

21. Ruzicka M, McCormick B, Magner P, Ramsay T, Edwards C, Bugela A, Hiremath S. Thiazide diuretic-caused hyponatremia in the elderly hypertensive: will a bottle of Nepro a day keep hyponatremia and the doctor away? Study protocol for a proof-of-concept feasibility trial. Pilot Feasibility Stud. 2018:4:71. https://doi.org/10.1186/s40814-018-0263-y. 
22. Westlund E, Stuart EA. The nonuse, misuse, and proper use of pilot studies in experimental evaluation research. Am J Eval. 2016;38(2):246-61. https:// doi.org/10.1177/1098214016651489.

23. Lee EC, Whitehead AL, Jacques RM, Julious SA. The statistical interpretation of pilot trials: should significance thresholds be reconsidered? BMC Med Res Methodol. 2014;14:41. https://doi.org/10.1186/1471-2288-14-41.

24. Arnold DM, Burns KEA, Adhikari NKJ, Kho ME, Meade MO. Cook DJ; for the McMaster Critical Care Interest Group. The design and interpretation of pilot trials in clinical research in critical care. Crit Care Med. 2009;37(suppl):S6974. https://doi.org/10.1097/CCM.0b013e3181920e33.

25. Sheehan JL, Winzeler-Merçay U, Mudie MH. A randomized controlled pilot study to obtain the best estimate of the size of the effect of a thermoplastic resting splint on spasticity in the stroke-affected wrist and fingers. Clin Rehabil. 2006;20(12):1032-7. https://doi.org/10.1177/0269215506071267.

26. Roberts C, Torgerson DJ. Baseline imbalance in randomised controlled trials. BMJ. 1999;319(7203):185. https://doi.org/10.1136/bmj.319.7203.185.

27. Chester R, Costa ML, Shepstone L, Cooper A, Donell ST. Eccentric calf muscle training compared with therapeutic ultrasound for chronic Achilles tendon pain - a pilot study. Man Ther. 2008;13(6):484-91. https://doi.org/1 0.1016/j.math.2007.05.014.

28. Garcia MK, Driver L, Haddad R, Lee R, Palmer JL, Wei Q, Frenkel M, Cohen L. Acupuncture for treatment of uncontrolled pain in cancer patients: a pragmatic pilot study. Integr Cancer Ther. 2014;13(2):133-40. https://doi. org/10.1177/1534735413510558.

29. Galantino ML, Callens ML, Cardena GJ, Piela NL, Mao JJ. Tai chi for wellbeing of breast cancer survivors with aromatase inhibitor-associated arthralgias: a feasibility study. Altern Ther Health Med. 2013;19(6):38-44. PMID: 24254037

30. Julious SA, Swank DJ. Moving statistics beyond the individual clinical trial: applying decision science to optimize a clinical development plan. Pharm Stat. 2005:4(1):37-46. https://doi.org/10.1002/pst.149.

31. Proschan MA. Sample size re-estimation in clinical trials. Biom J. 2009;51(2): 348-57. https://doi.org/10.1002/bimj.200800266.

\section{Publisher's Note}

Springer Nature remains neutral with regard to jurisdictional claims in published maps and institutional affiliations.

Ready to submit your research? Choose BMC and benefit from:

- fast, convenient online submission

- thorough peer review by experienced researchers in your field

- rapid publication on acceptance

- support for research data, including large and complex data types

- gold Open Access which fosters wider collaboration and increased citations

- maximum visibility for your research: over $100 \mathrm{M}$ website views per year

At $\mathrm{BMC}$, research is always in progress.

Learn more biomedcentral.com/submissions 